\title{
Digit-all: Rare Diseases
}

Authors:

${ }^{*}$ Gareth Baynam, ${ }^{1-7}$ Lynsey Chediak, ${ }^{8}$ Gemma Bilkey, ${ }^{1,9}$

Dylan Gration, ${ }^{4}$ Samuel Agyei Wiafe ${ }^{3}$

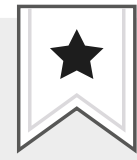

1. Office of Population Health Genomics, Public and Aboriginal Health Division, Perth, Australia

2. Department of Health, Government of Western Australia, Perth, Australia

3. Rare Disease Ghana Initiative, Accra, Ghana

4. Western Australian Register of Developmental Anomalies, King Edward Memorial Hospital, Perth, Australia

5. Telethon Kids Institute and Division of Paediatrics, Faculty of Health and Medical Sciences, University of Western Australia, Perth, Australia

6. Spatial Sciences, School of Earth and Planetary Sciences, Curtin University, Perth, Western Australia

7. Faculty of Medicine, Notre Dame University, Fremantle, Australia

8. World Economic Forum, Precision Medicine Initiative, San Francisco, USA

9. Patient Safety and Clinical Quality, Clinical Excellence Division, Department of Health, Government of Western Australia, Perth, Australia

*Correspondence to gareth.baynam@uwa.edu.au

Disclosure: $\quad$ The authors have declared no conflicts of interest.

Received: $\quad 28.07 .20$

Accepted: $\quad 21.08 .20$

Keywords: $\quad$ Digital health, phenotyping, precision medicine, precision public health, rare diseases, undiagnosed diseases.

Citation: $\quad$ EMJ. 2020;5[3]:11-16.

\section{Abstract}

Rare diseases are increasingly recognised as a global public health priority and contribute to significant and disproportionately high health system impacts. Accordingly, they present clinical and public health challenges, as well as opportunities for digital health solutions across the lifespan, including improved diagnosis, treatment, navigation and care coordination, and integration and coordination for broader societal and patient wellbeing. People living with rare diseases, individually and cumulatively, are digital disruptors. In this manuscript the authors describe some of the unique dynamics of the rare disease domain as they currently, or have the potential to in the future, apply to digital health; highlight some recent international rare diseases digital health initiatives; and touch upon implications for those with more common disorders.

\section{INTRODUCTION}

Rare diseases are increasingly recognised as a global public health priority. Whilst rare diseases have a low prevalence individually, it is estimated that the combined prevalence is between 6 and $8 \%$ of the population, ${ }^{2}$ equating to $>400$ million people globally. Most rare diseases

have a genetic association, and are often severely debilitating, impair physical and mental abilities, and shorten life expectancy. ${ }^{3}$ Rare diseases also contribute to significant and disproportionately high health system impacts, such as cost burden. ${ }^{4}$ These characteristics present clinical and public healthchallenges, as wellasopportunitiesfor digital health solutions across the lifespan, including 
improved diagnosis, treatment, navigation and care coordination, and integration and coordination for broader societal and patient wellbeing (e.g., linkage to education, disability, and community sectors). In this article, the authors describe some dynamics of the rare disease domain as they currently, or have the potential to in the future, apply to digital health; highlight some recent digital health initiatives in the international rare diseases domain; and touch upon implications for those with more common disorders.

\section{SO, WHAT IS DIGITAL?}

\section{Some Useful Definitions}

Digital is defined as recording or storing information as a series of the numbers 1 and $\mathrm{O}$, to show that a signal is present or absent; using or relating to digital signals and computer technology. ${ }^{5}$

"Digital health harvests data, information, and knowledge in real time from all societal activities, not just interactions with the health system and/ or data traditionally regarded as 'health' data; uses sophisticated analytics to distil knowledge from these data; intervenes in the widest possible range of societal and economic activities and technologies to encourage and generate better health and better value for health investments." ${ }^{6}$

Digital health systems are not just about health and health system workflows, they are about life and life-flows. Comprehensive digital health systems extend beyond illness into other areas of the patient's life. That is, the flows extend across the breadth of human experience to include a person's health, family, education, (dis)ability, economic (dis)advantage, and the community to which they belong. They require real-world data, information, and knowledge in real time, from all societal activities, to generate better health value. They also require approaches that are person-centric, decentralised, or distributed, and empower healthcare providers to actively participate and partner with each other and their patients, not simply to control a fixed outcome.

\section{WHY DIGITAL HEALTH AND RARE DISEASES?}

In addition to the very significant patient needs, there are a number of other dynamics of the rare diseases domain that make it fecund for digital health advances and implementation.

\section{Digital People}

Notwithstanding the complexity, fundamental humanity, and multidimensionality of living with a rare disease, rare monogenetic (single gene) diseases may be as close to a causally binary chronic disorder that medicine offers. That is, the presence of a single causative factor, for instance a mutation in one's DNA, can invariably (complete penetrance) or often (incomplete penetrance) lead to a manifest disorder. Put another way, a disruption in a digital (DNA) code can invariably or often result in the presence (1) or absence (0) of a chronic and severe condition. People living with rare diseases can be thought of as living digital disruptors; their diseases can have a binary model and they can shift the fundamental expectations and behaviours in a culture, market, industry, technology, or process that is caused by, or expressed through, digital capabilities, channels, or assets.

\section{Signal-to-Noise Ratio}

Signal-to-noise ratio is a measure used in science and engineering that compares the level of a desired signal to the level of background noise. A signal is a meaningful input whereas noise is a meaningless or unwanted input. The severity of rare diseases provides a high signal that is primed for a (digital) readout. The high signalto-noise ratios found in rare diseases are caused by the magnitude of both individual features of rare disorders and other unusual signatures of the condition itself that can be readily detected. These signals are also grounded in molecular biological pathways to provide insights into disease pathogenesis and its management. When combined, these factors provide clarity from the extremity of rare diseases. Similarly, the magnitude, and sometimes the relative speed of impact from an intervention, may be overt, which allows timely and effective monitoring. Accordingly, William Harvey (1578-1657), who was the first to describe the systemic circulation 
of blood pumped to the brain and body by the heart, noted: "Nature is nowhere accustomed more openly to display her secret mysteries than in cases where she shows traces of her workings apart from the beaten path; nor is there any better way to advance the proper practice of medicine than to give our minds to the discovery of the usual law of nature by the careful investigation of cases of rarer forms of disease. For it has been found in almost all things, that what they contain of useful or of applicable nature, is hardly perceived unless we are deprived of them, or they become deranged in some way."7

\section{Multisystemic}

Cumulatively, rare diseases affect all body systems, and individually they are often present with multisystem features. They traverse all medical specialities and the life-course. They are also exemplars for systems biology and the implementation of multi-omic approaches (i.e., combinations of genomics, epigenomics, phenomics etc). Because their individual rarity is combined with many layers of common elements, they require and are uniquely suited to cross-border, multi-health system care. Rare diseases are medically multisystemic, require systems biology, and health systems approaches that adapt to the flows of life.

\section{Runs on the Board}

The dynamics of rare diseases have already supported the implementation of digital health approaches in genomic healthcare, medical imaging and deep phenotyping, and international data sharing and matchmaking. ${ }^{8}$

\section{Healing Hands}

Caring for people with rare diseases requires combining traditional and physical approaches with modern technology. Put another way, there is a need to meld high-touch digital (the digits of healing hands) and high-tech digital (technology) approaches.

\section{WHAT'S HAPPENING IN DIGITAL HEALTH FOR RARE DISEASES?}

Increasingly, and particularly over the last 2 years, there has been global engagement and convergence in digital health for rare diseases. Some notable examples are touched upon below.

\section{Rare Diseases International and the World Health Assembly}

The $23^{\text {rd }}$ May 2019 marked a historic day for the rare diseases movement, with rare diseases featuring on the agenda of the World Health Assembly (WHA) for the first time. ${ }^{9}$ Rare Diseases International (RDI) contributed towards the formal and informal events that shone a spotlight on the importance of including rare diseases in universal health coverage in order to leave no one behind. At these events it was noted that rare diseases are at the forefront of digital health and they exemplify the high added value of regional and global approaches. They also showed that new digital tools are already being used to address the challenges experienced by the $>400$ million people living with a rare disease, enabling them to connect highly isolated patients, enable access to and acceleration of diagnosis, refer to specialised medical expertise, gather and share expertise on highly complex care, and advance clinical research.

A formal side event, part of the official WHA agenda and sponsored by permanent missions from the European Union, Romania, and Kuwait, used rare disease case studies to highlight the potential of digital health to achieve universal health coverage. The event was co-sponsored by an additional nine Member States, demonstrating the high level of interest and support.

\section{The Global Commission to End the Diagnostic Odyssey for Children with a Rare Disease}

The Commission is a global approach to accelerate time to diagnosis for children living with rare diseases. ${ }^{9}$ It uses a multidisciplinary group of international experts to develop a road map to accelerate the time to diagnosis for children living with rare diseases. They also develop, deploy, harmonise, and interoperate digital tools developed through pilots that traverse, but are not limited to, harnessing the combined power of engagement and awareness, genomics, deep phenotyping, and artificial intelligence, whilst equitably scaling their implementation. One digital tool that is currently being implemented in the Global Commission data ecosystem is Cliniface. ${ }^{10}$ This 3-dimensionsal facial visualisation and analysis software enables collaboration between clinicians and researchers to advance understanding of facial 
characteristics and their relationship with rare diseases and their treatment.

\section{World Economic Forum Precision Medicine Initiative}

Precision medicine can be defined as an emerging approach for disease treatment and prevention that takes into account individual variability in genes, environment, and lifestyle for each person..$^{10}$ Digital health tools are key enablers of precision medicine. The World Economic Forum (WEF) Precision Medicine Initiative operates in the context of enabling global public-private co-operation and committing to developing principles and frameworks that accelerate the application of science and technology for global public interest, whilst also mitigating for any potential risks of new personalised medicine applications in genomics or big data."

The Initiative focusses on two exemplar implementation domains: rare diseases and cancer. In order to realise the full potential of precision medicine (including related digital health approaches), substantial economic, regulatory, social, and technical challenges to its broad implementation must be overcome. Those identified through the WEF include: 1) generating sufficient evidence; 2) tackling data sharing and infrastructure challenges; 3) reshaping the regulatory environment; 4) adoption of genomic information from research into clinical care; 5) economics of precision medicine; 6 ) creating payment models involving the gains and risks shared along the value chain; and 7) attaining greater patient and clinician engagement and trust. The WEF supports pilot projects and other initiatives to address, and hopefully relieve, these bottlenecks. One such project is Lyfe Languages. ${ }^{12}$ Lyfe Languages is empowering and retaining Indigenous languages, creating more connected communities, and supporting equitable advances in digital healthcare. It is a community engaged and co-designed initiative to deliver Indigenous language translations of the lingua franca of precision phenotyping (the Human Phenotype Ontology [HPO]). Through these HPO translations, Lyfe Languages digitises and makes computer-readable descriptions of a condition's manifestations (the phenotype) that are provided directly in an individual's own Indigenous language.

\section{Capturing Phenotype Through the Life and Health System Journey}

In medicine, phenotype is a deviation from normal form, function, or behaviour. Phenotype can also be thought of as the voice of the patient and the clinicians describing that patient's condition, statically and dynamically. Tools such as Phenotips, ${ }^{13}$ Patient Archive, ${ }^{14}$ and Dx2915 have been developed to facilitate phenotyping, primarily for diagnostic support for rare diseases. Another example is Track.health, ${ }^{16}$ which delivers approaches to measure, monitor, and track a patient's journey within the health system, from start to finish.

\section{Primary Care}

A patient's medical journey starts and is often housed in primary care. As such, digital health integration within and between primary and specialist care is critical; this is especially true for complex conditions like rare diseases. The UK National Health Service (NHS) Long Term Plan states that every patient will be able to access a digital-first primary care offer by $2023 / 2024 .^{17}$ This, and other primary care initiatives, will need to embrace the challenges and opportunities of caring for people with rare diseases. The norm for people with rare diseases carrying large binders with their medical history and past appointment documentation in paper records can be significantly alleviated by a digital approach.

Other markers to embrace in digital health for rare diseases are: the proposed Innovative Medicines Initiative (IMI) call for newborn screening and digital health tools:; ${ }^{18}$ conferences that focus on digital health and rare diseases, such as the World Orphan Drug Congress ${ }^{19}$ and multiple other digital health initiatives for rare diseases launched by various pharmaceutical companies; and the IMI digital collaboration on rare diseases in Germany. ${ }^{20}$

\section{COMMON THEMES AND NEEDS}

Amongst the various rare diseases digital health initiatives there are common themes and requirements. Some of these include: 1) the need for patient engagement and co-design; 2) developing and implementing global technology standards in genotyping, phenotyping (e.g., Phenopackets, ${ }^{21}$ a developing open standard for 
sharing disease and phenotype information), and diagnostic coding (e.g., ORPHAcodes ${ }^{22}$; "The beginning of wisdom is to call things by their proper name");23 3) interoperability, including avoiding 'supersiloes' of patient and clinical/health system data; 4) a focus on equity and tools that can be employed in low-resource environments; 5) real-world data and patientcentred metrics; and 6) global connectivity that can be adapted to suit jurisdictional requirements. Fortunately, the rare diseases community is already heavily invested in many of these elements and is often at the forefront of addressing these issues.

\section{WHY DIGIT-ALL?}

Rare diseases can cross all medical specialties, across all the lifespan, all aspects of life, and all of the globe. Serving the unmet needs of people living with rare diseases requires all of us. All of the various stakeholders can be digitally connected for more timely, scalable, and sustainable digital health change. It also requires a focus that draws upon and empowers all of the community. Time and time again, discoveries in the rare diseases domain are translated to added benefits for people with more common disorders. ${ }^{24}$ For instance, one in two new medicines come from rare diseases research; for example, the cholesterol-lowering drugs, statins, are being developed as a result of researching the rare disease, familial hypercholesterolaemia. ${ }^{24}$ Specific digital health examples of solutions generated for rare diseases that have then been adapted to serve more common diseases include the pivot of Lyfe Languages from an initial focus on rare diseases to subsequently include the development of resources for novel coronavirus disease-2019 (COVID-19) and immunodeficiency; and the development of the COVID-19 symptom tracker, Covidaware.me, ${ }^{25}$ which is based on the rare diseases patient-facing knowledge aggregator rareaware.me. ${ }^{26}$ Serving the digital health needs for people living with rare diseases will benefit all of us.

\section{CONCLUSION}

Rare diseases present a global health challenge with high unmet need. Serving this need will provide opportunities to develop solutions for both rare and common diseases. The context of the challenges and the opportunities continue to evolve, and this is particularly evident in the field of digital health.

\section{References}

1. European Organisation for Rare Diseases (EORD). Rare diseases: understanding this public health priority. 2005. Available at: https:// www.eurordis.org/IMG/pdf/princeps document-EN.pdf. Last accessed: 24 August 2020.

2. Aymé S, Rodwell C. Report on the state of the art of rare disease activities in Europe. Orphanet J Rare Dis. 2012;7(Suppl 2):A1.

3. Schieppati A et al. Why rare diseases are an important medical and social issue. Lancet. 2008;371(9629):203941.

4. Walker CE et al. The collective impact of rare diseases in Western Australia: an estimate using a population-based cohort. Genet Med. 2017;19: 546-52.

5. Cambridge Online Dictionary. Meaning of digital in English. 2020. Available at: https://dictionary. cambridge.org/dictionary/english/ digital. Last accessed: 2 May 2020.

6. Rowlands D. What is digital health? and why does it matter? 2019.

Available at: https://www.hisa.org. au/wp-content/uploads/2019/12/

What_is_Digital_Health.pdf? $\times 97063$.

Last accessed: 13 May 2020.

7. Dooms MM. Rare diseases and orphan drugs: 500 years ago. Orphanet J Rare Dis. 2015;10:161

8. Philippakis AA et al. The Matchmaker Exchange: a platform for rare disease gene discovery. Hum Mutat. 2015;36(10):915-21.

9. Rare Diseases International. Rare diseases feature for firsttime at World Health Assembly. 2020. Available at: https://www. rarediseasesinternational.org/ rare-diseases-feature-for-first-timeat-world-health-assembly/. Last accessed: 24 August 2020.

10. Genetics Home Reference (GHR). What is precision medicine? 2020 Available at: https://ghr.nlm.nih.gov/ primer/precisionmedicine/definition. Last accessed: 24 August 2020.
11. World Economic Forum. Precision Medicine. 2020. Available at: https:// www.weforum.org/communities/ precision-medicine. Last accessed: 24 August 2020

12. Lyfe Languages. Human phenotype ontology - rare and genetic diseases. Available at: http://www. lyfelanguages.com/About.html. Last accessed: 2 May 2020

13. Girdea M et al. PhenoTips: patient phenotyping software for clinical and research use. Hum Mutat. 2013;34(8):1057-65.

14. Garvan Institute of Medical Research. "The best tool of its kind in the world": patient archive being used to advance diagnosis of rare diseases in WA. 2017. Available at: https:// www.garvan.org.au/news-events/ news/201cthe-best-tool-of-its-kindin-the-world201d-patient-archivebeing-used-to-advance-diagnosis-ofrare-diseases-in-wa. Last accessed: 2 May 2020. 
15. Foundation 29. Dx29. 2020. Available at: https://www.dx29.ai/. Last accessed: 2 May 2020.

16. Track.health. Measure, monitor and manage your patient's journey from start to finish. 2019. Available at: https://track.health/. Last accessed: 2 May 2020.

17. National Health Service (NHS). The NHS long term plan - Chapter 5: digitally-enabled care will go mainstream across the NHS. 2019. Available at: https://www. longtermplan.nhs.uk/online-version/ chapter-5-digitally-enabled-care-willgo-mainstream-across-the-nhs/. Last accessed: 13 May 2020.

18. European Commission (EC). Shortening the path to rare disease diagnosis by using newborn genetic screening and digital technologies. 2020. Available at: https://ec.europa.eu/info/fundingtenders/opportunities/portal/screen/ opportunities/topic-details/imi22020-23-05. Last accessed: 13 May 2020

19. Terrapinn. World Orphan Drug Congress - Digital Health \& Artificial Intelligence. 2020. Available at: https://www.terrapinn.com/ conference/world-orphan-drugcongress-usa/Digital-Health-ArtificialIntelligence.stm. Last accessed: 13 May 2020.

20. Medical Informatics Initiative Germany. Digital collaboration on rare diseases. 2020. Available at: https:// www.medizininformatik-initiative.de/ en/digital-collaboration-rare-diseases. Last accessed: 13 May 2020.

21. Phenopackets. Open and computable bioinformation. 2016. Available at: http://phenopackets.org/. Last accessed: 2 May 2020.

22. Orphadata. Rare Diseases and Classifications. Available at: http:// www.orphadata.org/cgi-bin/rare_free. html. Last accessed: 2 May 2020

23. Wikiquote. Confucius. 2020. Available at: https://en.wikiquote.org/wiki/ Confucius. Last accessed: 13 May 2020.

24. Gahl WA. The battlefield of rare diseases: where uncommon insights are common. Sci Transl Med. 2012;4(154):1-3.

25. Covidaware.me. 2020. Available at: https://covidaware.me/. Last accessed: 25 August 2020.

26. Rareaware.me. 2020. Available at: https://rareaware.me/. Last accessed: 25 August 2020. 